\title{
Comparison of Phage-Based Magnetoelastic Biosensors with Taqman- Based Quantitative Real-Time PCR for the Detection of Salmonella typhimurium Directly Grown on Tomato Surfaces
}

Mi-Kyung Park ${ }^{1 *}$, Jang Won Park ${ }^{2}$, Howard C. Wikle, III ${ }^{1}$ and Bryan A. Chin ${ }^{1}$

${ }^{1}$ Materials Research and Education Center, Auburn University, 275 Wilmore Labs, Auburn, Alabama, 36849, USA

${ }^{2}$ Simmons Comprehensive Cancer Center, University of Texas Southwestern Medical Center, 5323 Harry Hines Blvd., Dallas, TX 75390 , USA

\begin{abstract}
A phage-based magnetoelastic (ME) biosensor method was compared with a TaqMan-based quantitative realtime PCR (Q-PCR) method for the detection of Salmonella typhimurium on tomato surfaces. This ME biosensor method utilizes magnetoelastic resonators coated with E2 filamentous phage to bind with and measure the concentration of S. typhimurium. In this study, standard curves, correlations, and limits of detection (LOD) for the ME biosensor and Q-PCR methods were determined by inoculating tomato surfaces with $S$. typhimurium suspensions in concentrations ranging from 1 to 8 log CFU/tomato. The LOD for the ME biosensor method and Q-PCR were 3 and 2 log CFU/tomato, respectively. In a direct comparison of the detection methods, S. typhimurium suspensions (3 log CFU/tomato) were inoculated on 65 tomato surfaces, then incubated at $37^{\circ} \mathrm{C}$ and $100 \% \mathrm{RH}$ for $24 \mathrm{~h}$. After $24 \mathrm{~h}, \mathrm{~S}$. typhimurium was positively detected by both methods and the quantified concentrations were nearly the same, $(6.35$ $\pm 2.03)$ and $(6.34 \pm 0.17) \log$ CFU/tomato respectively for the ME biosensor method and the Q-PCR method, which were significantly greater than the concentration determined by the BGS-plate count method $(5.33 \pm 0.21)$. Scanning electron microscopy (SEM) was used to confirm the growth of $S$. typhimurium on the tomato surfaces and the binding of $\mathrm{S}$. typhimurium on the measurement sensors. This study demonstrated that the ME biosensor method was robust and competitive with Q-PCR for S. typhimurium detection on fresh produce.
\end{abstract}

Keywords: Salmonella typhimurium; Magnetoelastic biosensor; E2 phage; Quantitative real-time PCR; Probe; Limit of detection

\section{Introduction}

Outbreaks of Salmonella infection have recently occurred in a wide variety of fresh fruits and vegetables including lettuce, tomatoes, alfalfa sprouts, alfalfa seeds, artichokes, bean sprouts, cabbage, cilantro, lettuce, parsley and tomatoes, etc. [2]. From 1996 to 2008, eighty-two foodborne illness outbreaks were associated with the consumption of fresh produce (FDA, 2009). Of these outbreaks, 14 outbreaks (17.1\%) were associated with the consumption of tomatoes and led to 1,927 illnesses and 3 deaths in the United States (FDA, 2009). Tomatoes have been one of the most common of fresh produce items implicated with Salmonella outbreaks [3]. Since contamination can occur at any point along the farm to fork continuum, it would be ideal for fresh tomatoes to be inspected systematically in the field or on the processing line with a real-time and on-site detection methods. Rapid and direct detection of Salmonella on fresh produce will decrease the number of illness outbreaks by providing timely data on the identification and quantification of Salmonella in contaminated foods [3].

Considerable effort has been directed towards the development of rapid and simple detection methods such as polymerase chain reaction (PCR), enzyme-linked immunosorbent assay (ELISA), and several types of biosensor methods in order to substitute for the laborious and time-consuming conventional detection methods [5,29,30]. PCR is one of most promising alternative methods of detecting Salmonella in foods since many studies have shown that PCR is well suited for rapid, sensitive, specific and accurate bimolecular detection $[5,11,16]$. In addition, recently emerging PCR assays with fluorogenic probes (dye SYBR green, TaqMan, molecular beacons, and scorpion) have facilitated the development of real-time PCR assays more accurate and sensitive towards the identification and quantification for Salmonella $[5,8,20]$. In a preliminary study, a TaqMan-based quantitative real-time PCR method (Q-PCR) for the detection of S. typhimurium on tomato surfaces was demonstrated. The TaqMan assay system consists of two primers and a probe that binds with a specific site of the PCR amplicon produced during the amplification process. As the PCR process continues, the 5' nuclease activity of DNA polymerase cleaves the probe resulting in separation of the reporter and quencher dyes. The cleavage of the probe increases the fluorescence and its intensity is proportional to the amount of PCR amplicon produced $[6,19]$.

A phage-based magnetoelastic (ME) biosensor has been developed as a novel, wireless, direct detection method for in-field use $[10,13,17,18,27]$. ME biosensors have been successfully demonstrated to detect pathogens and spores such as S. typhimurium and Bacillus anthracis. The phage-based ME biosensor is composed of a ME resonator platform coated with genetically engineered filamentous phages for the specific recognition of a target pathogen. The detailed principle of ME biosensor operation has been explained in previous studies $[18,23,24]$. In brief, the ME biosensor method is based on measuring the change in resonant frequency which is proportional to the number of $S$. typhimurium cells bound to the ME resonator platform. The ME resonator platform is made from a magnetostrictive material that elongates or contracts under an applied external magnetic field. The platform's resonant frequency is measured wirelessly using an

*Corresponding author: Mi-Kyung Park, Materials Research and Education Center, Auburn University, 275 Wilmore Labs, Auburn, Alabama, 36849, USA, Tel: +1 334844 7443; Fax +1 334844 3400; E-mail: parkmik@auburn.edu

Received October 25, 2011; Accepted December 09, 2011; Published December 14, 2011

Citation: Park MK, Park JW, Wikle HC III, Chin BA (2012) Comparison of PhageBased Magnetoelastic Biosensors with Taqman-Based Quantitative Real-Time PCR for the Detection of Salmonella typhimurium Directly Grown on Tomato Surfaces. J Biosens Bioelectron 3:113. doi:10.4172/2155-6210.1000113

Copyright: (C) 2012 Park MK, et al. This is an open-access article distributed under the terms of the Creative Commons Attribution License, which permits unrestricted use, distribution, and reproduction in any medium, provided the original author and source are credited. 
Citation: Park MK, Park JW, Wikle HC III, Chin BA (2012) Comparison of Phage-Based Magnetoelastic Biosensors with Taqman-Based Quantitative Real-Time PCR for the Detection of Salmonella typhimurium Directly Grown on Tomato Surfaces. J Biosens Bioelectron 3:113. doi:10.4172/2155-6210.1000113

inductive pick-up coil. On binding with S. typhimurium, the mass of the ME biosensor platform increases resulting in a decrease in the platform's resonant frequency. Therefore, S. typhimurium can be identified and quantified by the resonant frequency changes in the phage-based ME biosensor. A recent study [23] demonstrated the practical suitability of the ME biosensor method by detecting $S$. typhimurium grown directly on the surface of tomatoes under favorable humidity and temperature conditions.

The comparison with other widely recognized alternative detection methods will be necessary in order to fully validate and evaluate the ME biosensor method. To date, no other investigation is known to have compared the ME biosensor method with a TaqMan-based quantitative real-time PCR (Q-PCR) method. Based on the validated set of primers and advances in Q-PCR assay, the detection limit of the phage-based ME biosensor method was compared with a Q-PCR analysis by developing linear standard curves after controlled growth of $S$. typhimurium on tomato surfaces. S. typhimurium was artificially inoculated then directly grown on tomato surfaces, detected, and the concentration quantified using the equations of the standard curves. Finally, the practicability and applicability of the ME biosensor method is compared with Q-PCR assay for use as an on-site and in-field detection method.

\section{Materials and Methods}

\section{Bacterial strains}

S. typhimurium (ATCC 13311) was provided from Dr. James M. Barbaree's Laboratory in the Department of Biological Sciences at Auburn University, Auburn, AL.

\section{Tomatoes and spot-inoculation}

Red, ripe tomatoes (Lycopersicon esculentum var. esculentum) were purchased from a local grocery store (Auburn, AL, USA). Only tomatoes free of visible detects such as bruises, cuts, and abrasions were used after washing with sterilized-distilled water (DW) five times to remove all possible contaminants. A square $\left(5 \times 5 \mathrm{~mm}^{2}\right)$ was drawn on the tomato surface with a fine-tip permanent marker using a sterile paper template for spot-inoculation. An aliquot of $10 \mu \mathrm{l}$ suspension was inoculated in the center of the marked area on the tomato surface. The inoculated tomato was placed in a glass container and was placed under laminar air flow for $90 \mathrm{~min}$ to allow the attachment S. typhimurium on the tomato surfaces.

\section{TaqMan-based quantitative real-time PCR (Q-PCR) methodology}

Primers and probe: PCR primers and probe for the TaqMan assay were originally designed by Park et al. (2008) [22] and the specificity of these primers were evaluated in a preliminary study. The primers were synthesized by IDT (Integrated DNA Technologies Inc., Coralville, IA, USA). The sequences of the primers were as follows: forward primer Sal-F, 5'-GCG CAC CTC AAC ATC TTT C-3' (Tm 54.4 ${ }^{\circ} \mathrm{C}$ ); and reverse primer Sal-R, 5'-CGG TCA AAT AAC CCA CGT TCA$3^{\prime}\left(\operatorname{Tm} 55.4^{\circ} \mathrm{C}\right)$. The probe was labeled with the fluorescent reporter dye 6-carboxyfluorescein (FAM) on the 5' end and with Minor Groove Binder-NonFluorescent Quencher (MGB-NFQ) on the 3' end. The sequence of the probe was follows: 6FAM ATC ATC GTC GAC ATG CMGBNFQ [22]. Primers and probe concentration were optimized using a 7300 Real-Time PCR System (Applied Biosystems, Foster City, CA, USA).
TaqMan-based quantitative real-time PCR (Q-PCR) protocol: Q-PCR was performed using the 7300 Real-Time PCR system. A reaction volume of $25 \mu \mathrm{l}$ of PCR mixture containing $12.5 \mu \mathrm{l}$ of TaqMan universal PCR master mix (Applied Biosystems, Foster City, CA), 10 $\mu \mathrm{M}$ each of primers and probe, filtered DW and $5 \mu \mathrm{l}$ of extracted DNA. The thermal cycling condition was: $2 \mathrm{~min}$ at $50^{\circ} \mathrm{C}, 10 \mathrm{~min}$ at $95^{\circ} \mathrm{C}, 50$ cycles of $15 \mathrm{sec}$ at $95^{\circ} \mathrm{C}$, and $1 \mathrm{~min}$ at $60^{\circ} \mathrm{C}$. All of the experiments were performed in duplicate or triplicate with positive and negative controls. Detection and quantification of amplified DNA was expressed as $\mathrm{Ct}$ values. If a Ct value exceeded 40 , this reaction contained no target DNA and was considered to be a negative amplification $[14,15]$.

\section{E2 phage-based magnetoelastic (ME) biosensor methodology}

Preparation of magnetoelastic strip-shaped resonator platforms and E2 phage immobilization: Magnetoelastic (ME) resonator platforms, $0.028 \mathrm{~mm} \times 0.2 \mathrm{~mm} \times 1 \mathrm{~mm}$ in size, were fabricated following the procedures described in a previous study [18]. E2 phage was provided in the form of a suspension $\left[1.0 \times 10^{12} \mathrm{vir} / \mathrm{ml}\right.$ in TrisBuffered Saline (TBS, pH 7.4)] by Dr. Barbaree's Laboratory. The E2 phage suspension was mixed with the equal amounts of TBS buffer to adjust the concentration of E2 phage $\left(5.0 \times 10^{11} \mathrm{vir} / \mathrm{ml}\right.$ in TBS). Each ME resonator platform was placed in an Eppendorf tube containing 300 $\mu \mathrm{l}$ of diluted E2 phage suspension and incubated on a rotary shaker $(8$ $\mathrm{rpm}$ ) at $22^{\circ} \mathrm{C}$ for $1 \mathrm{~h}$. The resonator platforms were then washed three times with the TBS buffer and twice with sterilized DW in order to remove any unbound phage and salt debris. After immobilization of E2 phage, any unbound area of the resonator platform was blocked with $300 \mu \mathrm{l}$ of $1 \%$ bovine serum albumin (BSA, Sigma-Aldrich Co., St. Louis, $\mathrm{MO}, \mathrm{USA}$ ) at $22^{\circ} \mathrm{C}$ for $1 \mathrm{~h}$. Finally, the resonator platform was washed three times with DW and allowed to air dry. The ME resonator platform was then ready for use as a measurement sensor. Control sensors were prepared using the same procedure, except for the elimination of the E2 phage immobilization step. The control sensors were used to compensate for the effects of environmental changes and non-specific binding. Prior to placement of both measurement and control sensors on an inoculated tomato surface, the initial resonant frequency of both sensors were measured with an HP 8751A network analyzer combined with an S-parameter test set.

ME biosensor protocol: The measurement and control sensors were placed on the tomato surfaces within the outlined areas. In order to increase the chance of making contact with S. typhimurium, three sets of measurement and control sensors were placed on the tomato surfaces. The tomatoes were placed in a humidity controlled container $(\mathrm{RH} 100 \%)$ and incubated at $30^{\circ} \mathrm{C}$ for $30 \mathrm{~min}$ [24]. After incubation, the measurement and control sensors were placed in the center of an inductive pick-up coil connected to the network analyzer. Finally, the resonant frequencies of the sensors were measured 10 times with the network analyzer which provided an average frequency shift data point. The resonant frequencies of the sensors before and after the placement on the tomato were compared $[23,24]$.

\section{Determination of limit of detection (LOD)}

Tomatoes were divided into two groups containing 27 tomatoes each for testing with ME biosensors and Q-PCR. For the spot-inoculation on the tomato surfaces, $10 \mu \mathrm{l}$ of $S$. typhimurium suspension, ranging from $3 \log \mathrm{CFU} / \mathrm{ml}$ to $10 \log \mathrm{CFU} / \mathrm{ml}$ in $0.03 \%$ TSB (Trypticase ${ }^{*}$ Soy Broth, Difco Laboratories, Sparks, MD, USA), were inoculated on the defined area following the procedures described in section 2.2. In each group, three tomatoes were inoculated with the same concentration of S. typhimurium. After inoculation, one group of tomatoes was analyzed 
Citation: Park MK, Park JW, Wikle HC III, Chin BA (2012) Comparison of Phage-Based Magnetoelastic Biosensors with Taqman-Based Quantitative Real-Time PCR for the Detection of Salmonella typhimurium Directly Grown on Tomato Surfaces. J Biosens Bioelectron 3:113. doi:10.4172/2155-6210.1000113

using the ME biosensor procedures described in section 2.4.2. For the other group, the inoculated area was cut from the tomato with a sterilized knife for Q-PCR analysis. The cuts were transferred to a centrifuge tube containing $1 \mathrm{ml}$ of sterilized phosphate buffered saline (PBS, pH 7.2, Sigma-Aldrich Co., St. Louis, Mo, USA) and glass beads. After mixing vigorously for $3 \mathrm{~min}$, the cut suspensions were centrifuged at $5000 \mathrm{~g}(7500 \mathrm{rpm})$ for $10 \mathrm{~min}$ and the precipitations were purified using DNeasy tissue kit (Qiagen, Hilden, Germnay) according to the manufacturer's instruction. Each purified DNA sample was diluted with filtered DW to a total volume of $625 \mu \mathrm{l}$ so that the volume fraction $(1 / 125)$ of one Q-PCR sample $(5 \mu \mathrm{l})$ corresponded to the same area fraction $(1 / 125)$ of one ME biosensor $(0.2 \mathrm{~mm} \times 1 \mathrm{~mm})$ over the same inoculation area $\left(5 \times 5 \mathrm{~mm}^{2}\right)$. In addition, interference by PCR inhibitors possibly existing in the food matrix would be reduced by the dilution $[25,29]$. The concentration of genomic DNA was measured with a Nanodrop spectrophotometer (Thermo Fisher Scientific Inc. Wilmington, DE, USA) and Q-PCR was performed in triplicate in order to create the standard curve. The amplification efficiency (AE) of Q-PCR was calculated following the formula $\mathrm{E}=\left(10^{-1 / \text { slope }}-1\right) \times 100$ [5] Lower AE means that the quantity of PCR products generated at each cycle decreases so that the amplification will be take longer.

\section{Application of ME biosensor and Q-PCR for S. typhimurium detection}

The viability of bacteria obtained from artificially inoculated samples may differ from that of naturally contaminated samples due to unfavorable growth conditions in nature [21]. Therefore, $S$. typhimurium was inoculated with $0.03 \%$ of TSB and grown on tomato surfaces in order to mimic natural environmental conditions. Tomatoes were divided into two groups containing 65 tomatoes in each group and with 5 tomatoes used for controls (inoculated with sterilized DW instead of cell suspension). An aliquot of $10 \mu \mathrm{l}$ of $S$. typhimurium suspension at $5 \log \mathrm{CFU} / \mathrm{ml}$ in $0.03 \%$ TSB medium was inoculated on tomato surfaces following the procedures described in section 2.2. The inoculated tomato samples were incubated at $37^{\circ} \mathrm{C}$ for $24 \mathrm{~h}$ with $100 \%$ $\mathrm{RH}$ in order to increase the number of $S$. typhimurium bacteria. One group of tomato samples was analyzed by the ME biosensor analysis and the other group by Q-PCR analysis using the procedures described in section 2.5 .

For enumeration of viable S. typhimurium, plate counting of cultured samples was performed. An aliquot of $10 \mu \mathrm{l}$ of $S$. typhimurium suspension at $5 \log \mathrm{CFU} / \mathrm{ml}$ in $0.03 \% \mathrm{TSB}$ was inoculated on the defined $5 \times 5 \mathrm{~mm}^{2}$ areas on 10 tomato surfaces and five tomatoes were inoculated with sterilized DW as a control experiment. All of tomatoes were incubated at the same conditions mentioned above. After incubation, the defined area on the tomato surface was cut with a sterilized knife and the cuts were transferred to a centrifuge tube containing $1 \mathrm{ml}$ of PBS and glass beads. After mixing vigorously for 3 min, $100 \mu \mathrm{l}$ of the mixture was diluted serially with PBS buffer and the serially diluted mixture was plated in triplicate on brilliant green sulfa (BGS) agar (Difco Laboratories, Sparks, MD, USA). Finally, the plates were incubated at $37^{\circ} \mathrm{C}$ for $24 \mathrm{~h}$ and the colonies were counted. The colony count results were divided by 125 to scale to the same fraction as previously employed and the result was expressed as log CFU/tomato.

\section{Microscopic analysis}

A JEOL-7000F scanning electron microscope (SEM) was used to confirm the attachment and growth of $S$. typhimurium on the tomato surfaces and binding of $S$. typhimurium to measurement and control sensors. A $10 \mu \mathrm{l}$ aliquot of S. typhimurium $(5 \log \mathrm{CFU} / \mathrm{ml}$ in $0.03 \%$
TSB) was inoculated on defined areas on ten tomato surfaces following the procedures described in section 2.2. For five of the tomatoes, the inoculated areas were cut from the tomato with a sterilized knife after attachment. The other five tomatoes were incubated at $37^{\circ} \mathrm{C}$ for $24 \mathrm{~h}$ with $100 \% \mathrm{RH}$ and then the surface was cut for SEM observation. In order to observe the $S$. typhimurium, the cuts were exposed to osmium tetroxide $\left(\mathrm{OsO}_{4}\right)$ vapor for $12 \mathrm{~h}$ to fix the bacteria followed by gold coating to a thickness of $140 \mathrm{~nm}$ using a PELCO sputter coater (Ted Pella Inc., Redding, CA, USA). In order to confirm the binding of S. typhimurium to the sensors, both measurement and control sensors were exposed to $\mathrm{OsO}_{4}$ vapor for at least $45 \mathrm{~min}$. The tomato segments and sensors were mounted on aluminum stubs for examination by SEM.

\section{Statistical analysis}

The experiments were replicated at least three times and the experimental results were expressed as arithmetic means (M) and standard deviations (SD). The student's paired t test for two groups and the one-way analysis of variance (ANOVA) among more than two groups were run to compare the means using GraphPad and InStat v.3 programs (GraphPad, San Diego, CA). The significance level was determined as $P<0.05, P<0.01$, or $P<0.001$. As a measure of repeatability, the variation coefficient $(\mathrm{CV})$ was calculated by the formula: $\mathrm{CV}=(\mathrm{SD} / \mathrm{M}) \times 100$.

\section{Results and Discussion}

In this study, we have compared our phage-based ME biosensor method with the well-known Q-PCR method. The specificity of both methods against $S$. typhimurium, one of the most important criteria to detect target cells, was evaluated in previous studies [22,28].

\section{Limit of detection (LOD) of Q-PCR}

The LOD in this study is defined as the lowest concentration of $S$. typhimurium that can be detected by Q-PCR or ME biosensor methods [7]. In Q-PCR, the threshold cycle $(\mathrm{Ct})$ value is defined as the point at which probe fluorescence rises appreciably above the background [29]. The measured Ct value corresponding to genomic equivalent of the logarithmic CFU is plotted in Figure 1. As shown in Figure 1, the $\mathrm{Ct}$ value decreased as the genomic equivalent of the logarithmic CFU increased. A linear relationship was found with a correlation coefficient $\left(R^{z}\right)$ of 0.977 and a generated slope of -3.456 . The amplification efficiency was $95 \%$ which was fit to the recommended range of 90 to 110\% (Applied Biosystems, 2008). Since the Ct average $(\mathrm{Ct}=44.213$ ) at $1 \log$ CFU/tomato was greater than 40, the LOD of Q-PCR assay was determined to be $2 \log \mathrm{CFU} /$ tomato for S. typhimurium detection. The LOD of Q-PCR was significantly lower than Guo et al's [11] study (2000), which showed that the LOD of PCR assay using hilA primers for S. Montevideo detection was $5 \log \mathrm{CFU} /$ tomato.

\section{Limit of detection (LOD) of ME biosensor}

Figure 2 shows the resonant frequency changes of ME sensors to different concentrations of $S$. typhimurium. As the concentration of $S$. typhimurium increased, the measured resonant frequency shift increased, as anticipated. SEM microscopy also confirmed that the binding of $S$. typhimurium on the ME resonant platform increased. The LOD of ME biosensor is defined as the point of intersection of two linear lines (measurement and control sensor) where the measurement sensor rises appreciably above the control sensor. As shown in Figure 2, the LOD for the ME biosensor method was determined to be $3 \log$ $\mathrm{CFU} /$ tomato. The resonant frequency shift of the measurement sensor could be distinguished from the control sensors at S. typhimurium 
Citation: Park MK, Park JW, Wikle HC III, Chin BA (2012) Comparison of Phage-Based Magnetoelastic Biosensors with Taqman-Based Quantitative Real-Time PCR for the Detection of Salmonella typhimurium Directly Grown on Tomato Surfaces. J Biosens Bioelectron 3:113. doi:10.4172/2155-6210.1000113

concentrations of $3 \log \mathrm{CFU} /$ tomato and greater. Although the LOD of the Q-PCR was one order lower than that of the ME biosensor, the LOD is a possible limiting factor for either method, especially when Salmonella contamination may occur with 1000 cells or less. Nonetheless, the fact that the LOD of the ME biosensor was only one order higher than that of the Q-PCR showed that the ME biosensor method is competitive to Q-PCR, considered to be one of the most sensitive detection methods known to date $[5,11,16]$

The resonant frequency change of the measurement sensors over the range of $S$. typhimurium concentrations from $3 \log \mathrm{CFU} /$ tomato is also plotted in Figure 2. A linear relationship is shown with a correlation coefficient $\left(\mathrm{R}^{2}\right)$ of 0.944 and a generated slope of 1671 , which was lower than that $\left(\mathrm{R}^{2}=0.977\right)$ of $\mathrm{Q}-\mathrm{PCR}$. The sensitivity of the $\mathrm{ME}$ biosensor method, defined as the slope of the calibration curve [27], was calculated to be $1173 \mathrm{~Hz} /$ decade.

\section{Observation and enumeration of $S$. typhimurium directly grown on the tomato surface}

Since the most predominant place for harboring Salmonella in or on tomatoes has been found to be the outer surface (82\%) [12], the ME biosensor method has been applied directly to the tomato surfaces without any further sample preparation. S. typhimurium was inoculated and grown directly on the tomato surfaces in order to compare the ME biosensor method with Q-PCR. Our previous study [24] showed that minimum initial inoculum size for the $S$. typhimurium growth on the tomato surface was determined to be $3 \log \mathrm{CFU} /$ tomato surface with diluted nutrients. These findings agreed with Wei et al.s [31] study (1995) in that S. Montevideo could grow with the help of nutrients (for example, in puncture wounds). Without any nutrient sources, the bacteria could only survive for several days on tomato surfaces at 20 and $25^{\circ} \mathrm{C}$. Thus, $\mathrm{S}$. typhimurium suspension ( $3 \log \mathrm{CFU}$ in $0.03 \% \mathrm{TSB}$ ) was inoculated on each of the tomatoes surfaces and incubated.

The attachment and growth of $S$. typhimurium was confirmed by SEM microscopic analysis (Figure 3). As shown in Figure $3 \mathrm{~A}$ and $3 \mathrm{~B}$, obtained from SEM microscopic analysis, the distribution of $S$.

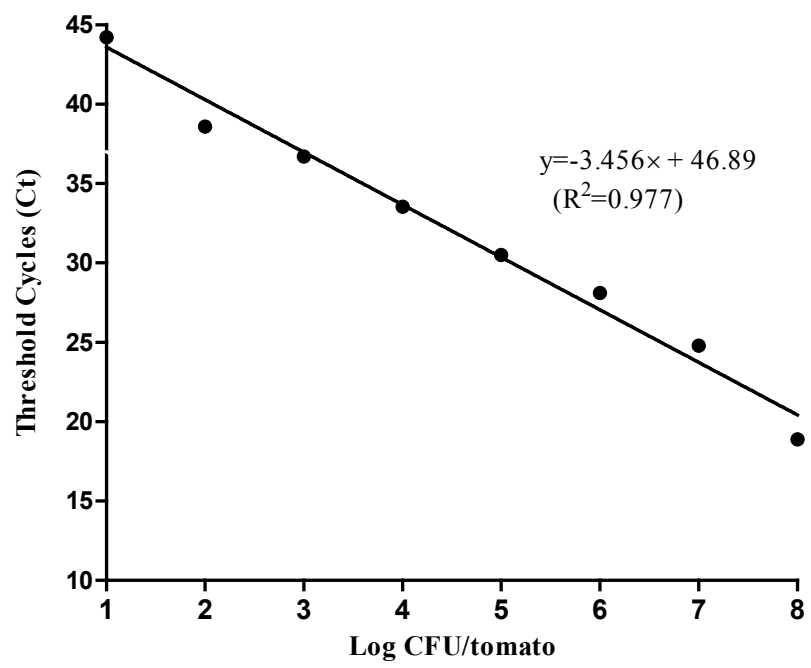

Figure 1: Standard curve for a 10-fold dilution series of $S$. typhimurium on tomato surfaces after Q-PCR performance. Threshold cycle $(\mathrm{Ct})$ is plotted on the axis of ordinates and log CFU genome equivalent is plotted on the axis of abscissae. A Ct value represents $1 / 125$ of the total inoculated area $\left(5 \times 5 \mathrm{~mm}^{2}\right)$, corresponding with the area of one ME sensor. Each data point represents the mean of triplicate samples taken from three replicate experiments $(n=9)$.

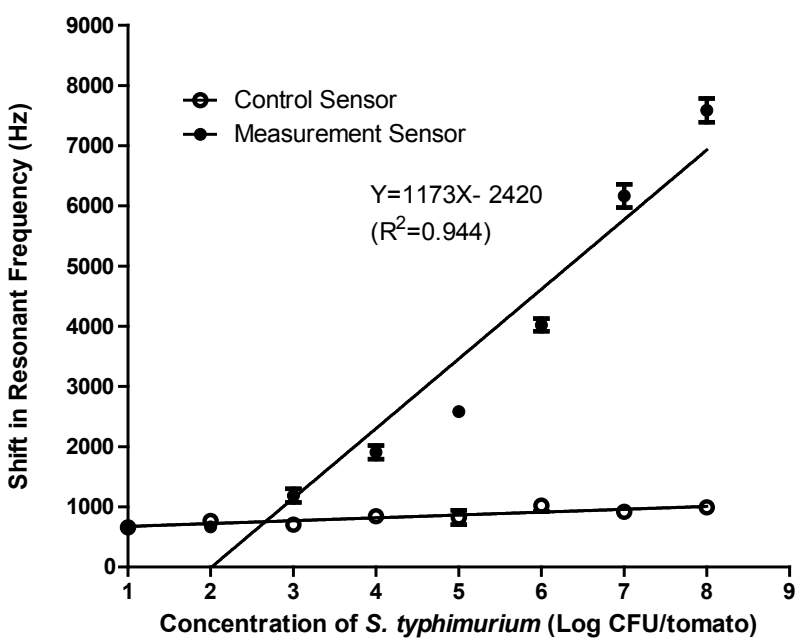

Figure 2: Resonant frequency shifts at various concentration of $S$. typhimurium inoculated on tomatoes surfaces. S. typhimurium was inoculated in a square area $\left(5 \times 5 \mathrm{~mm}^{2}\right)$ on tomato surfaces. A resonant frequency shift value represents $1 / 125$ of total inoculated area, corresponding with the area of one ME sensor. Each data point represents the mean of triplicate samples taken from three replicate experiments $(n=9)$.

typhimurium was not uniform before incubation. The number of $S$. typhimurium increased after the $24 \mathrm{~h}$ incubation and expanded to adjacent surfaces outside the inoculation area (Figure 3C, D). After incubation, the distribution of $S$. typhimurium was again found to be not uniform. The number of viable $S$. typhimurium was determined to be $5.33 \pm 0.21 \log$ CFU/tomato using the BGS-plate count method and this result was compared with the results obtained from the ME biosensor and Q-PCR.

Detection and quantification of S. typhimurium directly grown on the tomato surface using Q-PCR

S. typhimurium directly grown on the surface of tomatoes was detected by Q-PCR and the result shown in Figure 4. As a control experiment, five tomatoes inoculated with only sterilized DW showed negative amplification with $\mathrm{Ct}$ values exceeding 40 (data not presented). However, all 65 tomatoes inoculated with S. typhimurium showed positive amplification with $\mathrm{Ct}$ values ranging between 23.2 (6.85 log CFU/tomato) and 26.4 (5.93 log CFU/tomato) (Figure 4). This difference may be attributed to the variation in the attachment and growth of the cells on the tomato surfaces. Since the repeatability represents the precision of the PCR method, the repeatability was determined from the overall mean and standard deviation obtained from Figure 4. The mean and standard deviation were $24.97 \pm 0.71$ and the variation coefficient $(\mathrm{CV})$ was calculated to be $2.8 \%$. The quantified concentration of $S$. typhimurium was determined to be $6.34 \pm 0.17 \mathrm{log}$ $\mathrm{CFU} /$ tomato using the averaged Ct value of all data points (24.97) in the equation generated by Figure 1 (Figure 6). The concentration of $S$. typhimurium obtained in this manner was approximately one log higher than the BGS-plate count method $5.33 \pm 0.21 \log$ CFU/tomato, which is in good agreement with Jung et al's [15] (2005) findings, because DNA was purified from both dead and live cells on the tomato surfaces [26].

Detection and quantification of $S$. typhimurium directly grown on the tomato surface using ME biosensor

As shown in Figure 3D, the distribution of S. typhimurium on the inoculated tomato surfaces was not uniform so that three sets of 
Citation: Park MK, Park JW, Wikle HC III, Chin BA (2012) Comparison of Phage-Based Magnetoelastic Biosensors with Taqman-Based Quantitative Real-Time PCR for the Detection of Salmonella typhimurium Directly Grown on Tomato Surfaces. J Biosens Bioelectron 3:113. doi:10.4172/2155-6210.1000113

Page 5 of 8

sensors were used in order to increase the chances of bacteria coming into contact with sensors. Figure 5 shows the distribution of measured frequency shifts of numerous measurement and control sensors after exposure to $S$. typhimurium on the tomato surfaces. Significant differences in the resonant frequency shifts between measurement and control sensors $(\mathrm{P}<0.001)$ occurred, although some of measurement sensors were not significantly different from control sensors. Due to the uneven distribution of S. typhimurium on the tomato surfaces, some of the measurement sensors showed comparatively low resonant frequency shifts. SEM images confirmed that there were obvious differences in the number of $S$. typhimurium bound to control (Figure 5A) and measurement sensors (Figure 5B, 5C). The magnitude of the resonant frequency shift is proportional to the mass of cells bound to the sensors, visually evident in Figure 5B and 5C. All 65 tomato samples inoculated with $S$. typhimurium showed significant increases in the resonant frequency shift based on the average of three measurement sensors per tomato $(P<0.05)$. On the contrary, five tomatoes inoculated with only sterilized DW did not show any significant resonant frequency shift with either measurement or control sensors in the control experiment (data not presented). The repeatability of ME biosensor was determined to be $47.5 \%$ from the overall mean and standard deviation obtained from Figure 5. Finally, the quantified concentration of $S$. typhimurium was determined to be $6.35 \pm 2.03 \log \mathrm{CFU} /$ tomato using the equation generated by standard curve of Figure 2 .

\section{Comparison with BGS plate count method}

Both the ME biosensor and Q-PCR methods were compared and also were compared with the BGS-plate count method to quantify the concentration of $S$. typhimurium grown on tomato surfaces (Figure 6). As shown in Figure 6, the negative control sample inoculated with sterilized DW did not show any growth of $S$. typhimurium on tomato surface. Since the PCR method cannot differentiate between live and dead cells [26], it may be assumed that the quantified concentration determined by Q-PCR may be greater than that obtained from the BGSplate count method. The quantified concentration of S. typhimurium determined by Q-PCR method (6.34 $\pm 0.17 \log$ CFU/tomato) was approximately one order higher than the BGS-plate count method $(5.33 \pm 0.21)$, which was significantly different $(P<0.05)$. However, the quantified concentrations of $S$. typhimurium determined by the ME biosensor method $(6.35 \pm 2.03 \log$ CFU/tomato) was also significantly different from that from BGS-plate count method but there was no significant difference between the Q-PCR and ME biosensor method. The fact that the quantified concentration determined by both methods was very similar, most likely indicates that the E2 phage on the measurement sensor did not differentiate between live or dead cells. While it is not anticipated that the E2 phage can differentiate between live or dead cells, further work is required to demonstrate this. More
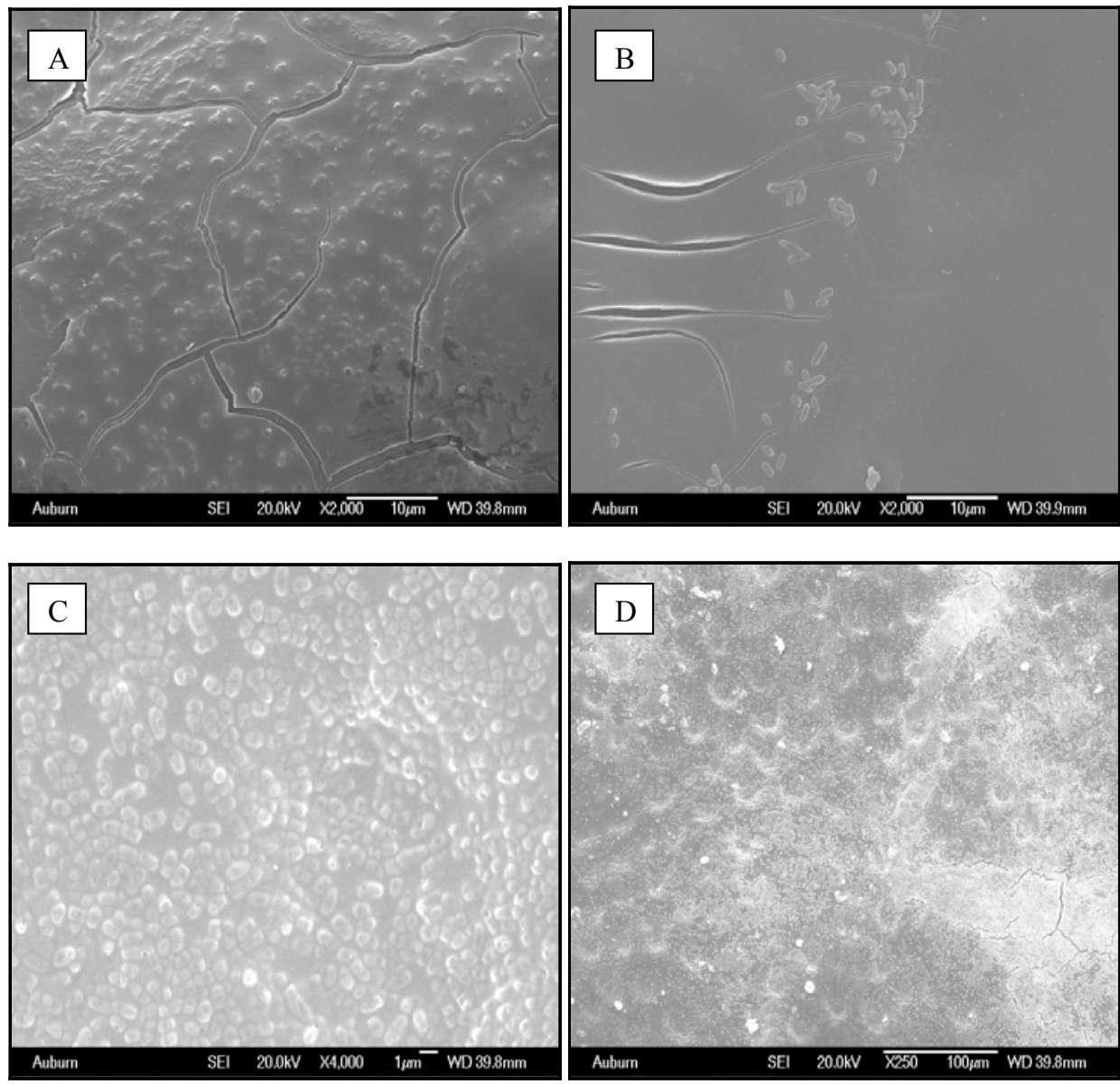

Figure 3: SEM images of S. typhimurium on the tomato surfaces; (A) (B) before and (C) (D) after 24-h incubation at $37^{\circ} \mathrm{C}$ with the initial concentration of 3.0 log CFU/tomato $\left(5 \times 5 \mathrm{~mm}^{2}\right)$. 
Citation: Park MK, Park JW, Wikle HC III, Chin BA (2012) Comparison of Phage-Based Magnetoelastic Biosensors with Taqman-Based Quantitative Real-Time PCR for the Detection of Salmonella typhimurium Directly Grown on Tomato Surfaces. J Biosens Bioelectron 3:113. doi:10.4172/2155-6210.1000113

Page 6 of 8

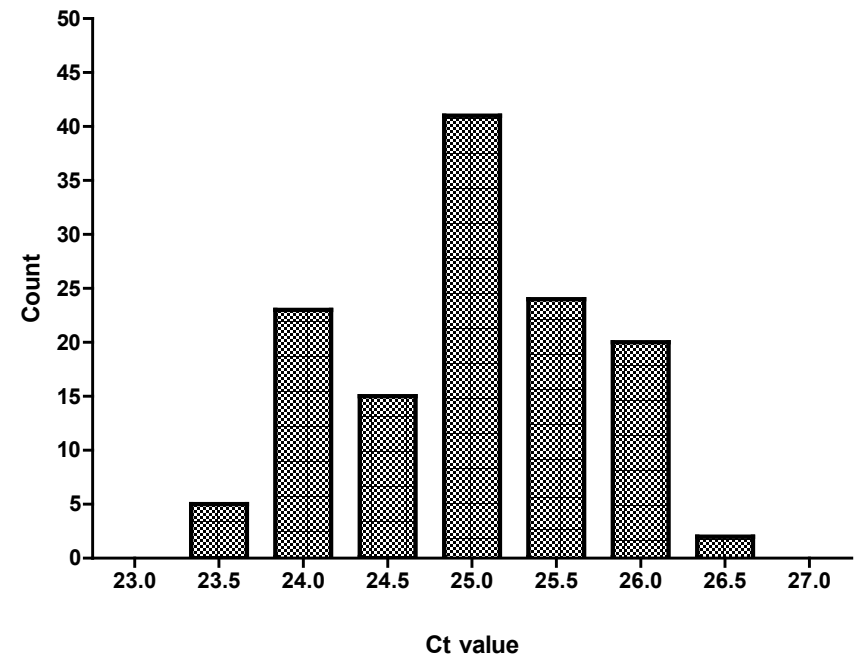

Figure 4: Distribution of $\mathrm{Ct}$ values for S. typhimurium detection using Q-PCR S. typhimurium (3 log CFU in $0.03 \%$ TSB medium) was inoculated on tomato surfaces $\left(5 \times 5 \mathrm{~mm}^{2}\right)$ and grown for $24 \mathrm{~h}$ at $37^{\circ} \mathrm{C}$ and $100 \% \mathrm{RH}$. Q-PCR was performed in duplicate for each tomato sample. A Ct value represents $1 / 125$ of total inoculated area $\left(5 \times 5 \mathrm{~mm}^{2}\right)$, corresponding with the area of one ME sensor.

importantly, this study showed that our ME biosensor method detected and quantified S. typhimurium grown on tomato surfaces with similar accuracy as Q-PCR.

\section{Comparison of practicability and applicability}

The direct detection of Salmonella or other bacteria without sample preparation using ME biosensors offers many advantages over competing methods, however, limitations may exist to the effectiveness of this method under certain conditions. Without sample preparation, one limitation to direct detection may be when S. typhimurium is found inside of the tomato in the juice or pulp, or when S. typhimurium is present under a firmly attached biofilm on the tomato surface. The mode of attachment of $S$. typhimurium to the tomato surface may also affect the accuracy of the ME biosensor method, depending on whether either specific or nonspecific adhesion to the tomato surface occurs which may be mediated by the bacterial cell structures such as pili or flagella. Another consideration is that the repeatability of the ME biosensor measurement (47.5\%) was found to be more susceptible to the uneven distribution of S. typhimurium on the tomato surfaces (Figure 3) than Q-PCR (2.8\%). The reduced opportunity for the bacteria and biosensor to interact at lower bacterial surface densities lessens the comparability of the ME biosensor and Q-PCR. When tomatoes with a more uniform distribution of bacteria were used, achieved by inoculating three tomato surfaces with $8 \log \mathrm{CFU} /$ tomato of $S$. typhimurium, the repeatability of the ME biosensor over a three day period was determined to be $5.7 \%$ (7732 \pm 442$)$. With a uniform distribution of S. typhimurium on the tomato surface, the repeatability of the ME biosensor method is both comparable and competitive with Q-PCR at 1.7\% (18.882 \pm 0.323$)$.

Despite these limitations, there are several advantages of the ME biosensor presented in this study compared to the practicability and applicability of Q-PCR. A previous study [4] showed that the structure

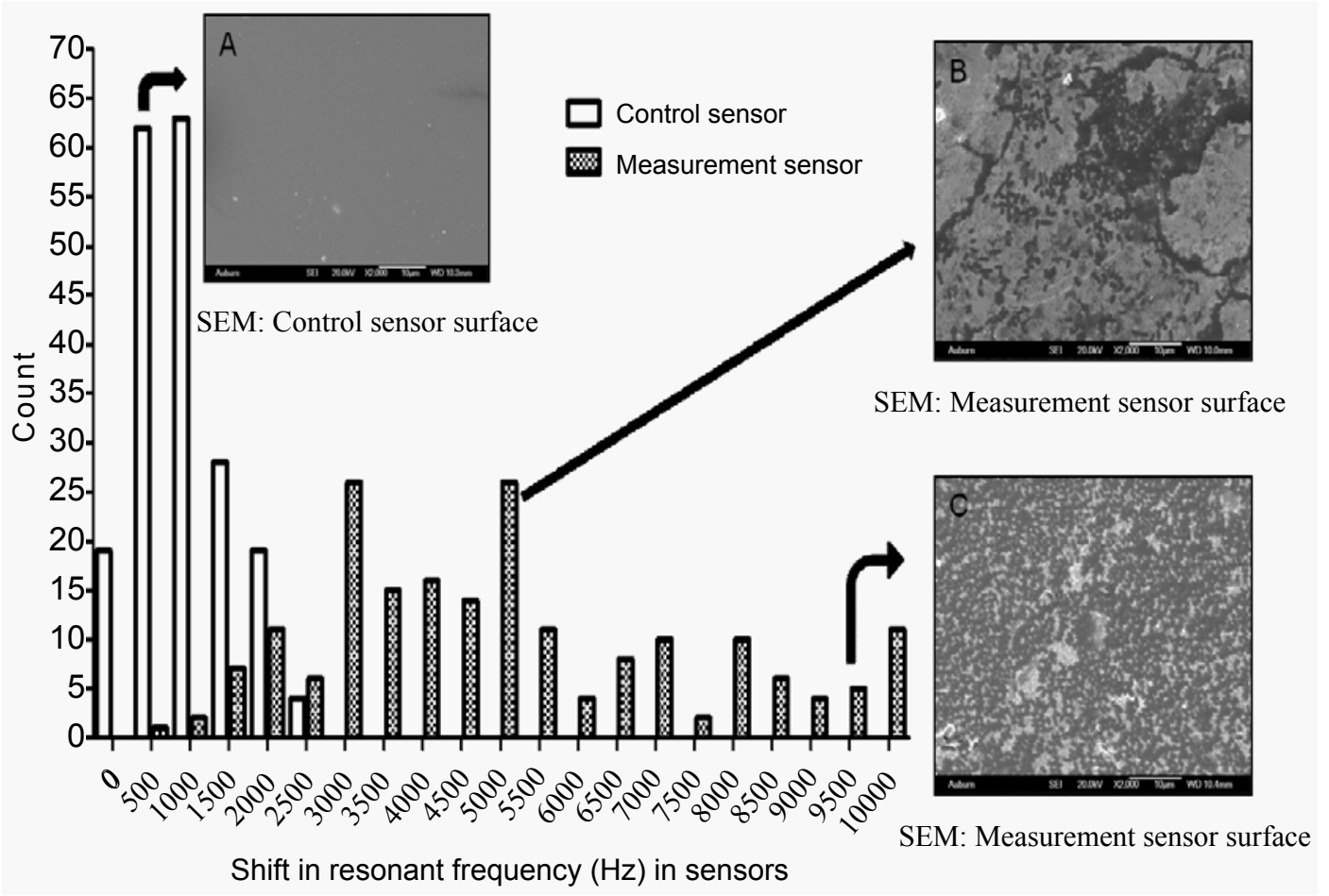

Figure 5: Distribution of resonant frequency shifts (left) and SEM images (A, B, C) of S. typhimurium detection using a phage-based ME biosensor method. $S$. typhimurium ( $3 \mathrm{log}$ CFU in $0.03 \%$ TSB medium) was inoculated on each of tomato surface $\left(5 \times 5 \mathrm{~mm}^{2}\right)$ and grown for $24 \mathrm{~h}$ at $37{ }^{\circ} \mathrm{C}$ and $100 \% \mathrm{RH}$. ME biosensor method was performed using three sets of measurement and control sensor per each tomato sample. A shift in resonant frequency was obtained from one sensor, corresponding with $1 / 125$ of total inoculated area $\left(5 \times 5 \mathrm{~mm}^{2}\right)$. 
Citation: Park MK, Park JW, Wikle HC III, Chin BA (2012) Comparison of Phage-Based Magnetoelastic Biosensors with Taqman-Based Quantitative Real-Time PCR for the Detection of Salmonella typhimurium Directly Grown on Tomato Surfaces. J Biosens Bioelectron 3:113. doi:10.4172/2155-6210.1000113

Page 7 of 8

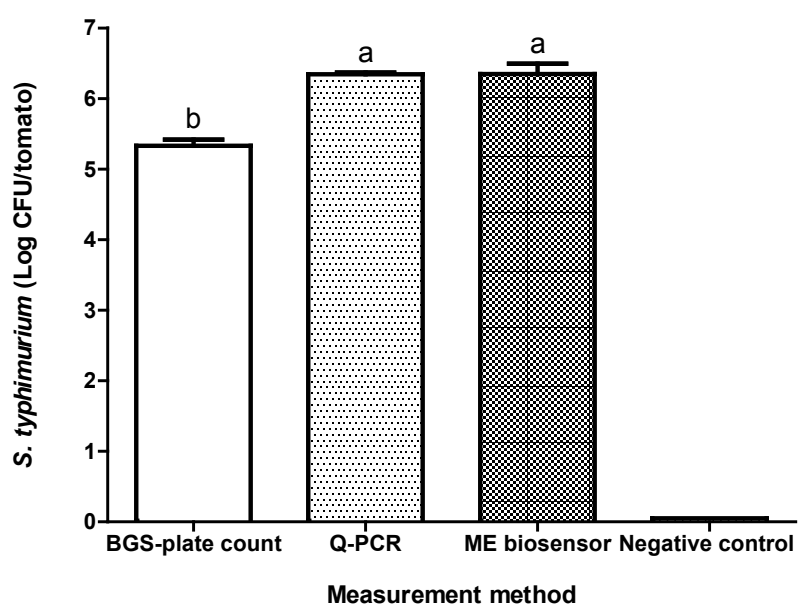

Figure 6: Concentration of $S$. typhimurium determined using BGS-plate count Q-PCR, and phage-based ME biosensor methods. S. typhimurium (3 log CFU in $0.03 \%$ TSB medium) was inoculated on each of tomato surface $\left(5 \times 5 \mathrm{~mm}^{2}\right)$ and grown for $24 \mathrm{~h}$ at $37{ }^{\circ} \mathrm{C}$ and $100 \% \mathrm{RH}$. The negative control was inoculated with sterilized DW and incubated for $24 \mathrm{~h}$ at $37^{\circ} \mathrm{C}$ and $100 \% \mathrm{RH}$ prio to BGS-plate count method. Different letters $(a, b)$ indicated that there were significant differences between measurement methods at $P<0.05$. Negative control was measured by both Q-PCR and ME biosensor method.

of E2 phage was very robust, even when exposed to harsh temperature conditions up to $80^{\circ} \mathrm{C}$. In another stability study of the $\mathrm{ME}$ biosensor method, the binding affinity of E2 phage with $S$. typhimurium was durable for two months even at $65^{\circ} \mathrm{C}$, with only a gradual decrease in the binding affinity observed with time [24]. Furthermore, the ME biosensor is a cost-effective detection method due to lack of costly instruments, DNA purification kits, and fluorescence probe and primers. Instead, the ME biosensor method requires only $1 \mathrm{~mm}$-size sensors, phage for a biorecognition probe, and relatively simple instrumentation for detection. In addition, the ME biosensor method is rapid, requiring only minutes of total detection time with no sample preparation involved whereas Q-PCR required 2-3 h detection times after sample preparation time. The level of training or expertise required to perform an analysis using the ME biosensor method is much less than Q-PCR method. Greater sensitivity (lower detection limit) can be obtained by decreasing the size of the ME biosensors [13]. By minimizing the sensor size and directly applying a large number of sensors to the fresh produce, broad coverage of fruits and vegetables can be obtained. This broader coverage is similar to increasing the number of samples taken, providing greater assurance that the produce is free of harmful levels of pathogens. These advantages contribute to the ME biosensor method being an effective on-site and in-field detection method to improve the food safety of fresh fruits and vegetables.

\section{Conclusions}

In this study, a phage-based ME biosensor method was compared with Q-PCR. The correlation coefficient and LOD for these two methods were calculated and determined to be 0.944 and $3 \log \mathrm{CFU} /$ tomato for the ME biosensor and 0.977 and $2 \log$ CFU/tomato for Q-PCR. When S. typhimurium was directly grown on tomato surfaces, both methods detected positively $S$. typhimurium on all 65 samples. The growth of $S$. typhimurium on the tomato surfaces and the binding of $S$. typhimurium on the measurement sensors for ME biosensor method was confirmed by SEM images. The quantified concentrations of $S$. typhimurium obtained using the equations developed from the standard curves were nearly the same for both methods. Although the LOD of the ME biosensor method was found to be one order more than that of Q-PCR, the ME biosensor method is robust and competitive with Q-PCR due to several advantages. The practicability and applicability of the ME biosensor method was demonstrated by detecting S. typhimurium within minutes without further tedious sample preparation. In addition, the $\mathrm{ME}$ biosensor detection method is cost-effective, simple to operate, and suitable for large-scale coverage. Thus, the ME biosensor method is a promising alternative to the Q-PCR method. This study demonstrates that the ME biosensor method has great potential to serve as an on-site and in-field detection method in order to improve the food safety of fresh produce.

\section{Acknowledgment}

This research was supported by the Auburn University Detection and Food Safety (AUDFS) Center with funding from the USDA-CSREES grant (204327 130851 2000)

\section{References}

1. Applied biosystems, 2008. Application note. Real-Time PCR: Understanding Ct Available at http://www3.appliedbiosystems.com/cms/groups/mcb_marketing/ documnts/generaldocuments/cms_053906.pdf (accessed 22 Dec 2010).

2. Beuchat, L.R., 2002. Microbes and Infection 4, 413-423.

3. Bisha B, Brehm-Stecher BF (2009) Simple adhesive-tape-based sampling of tomato surfaces combined with rapid fluorescence in situ hybridization for Salmonella detection. Appl Environ Microb 75: 1450-1455.

4. Brigati JR, Petrenko VA (2005) Thermostability of landscape phage probes Anal Bioanal Chem 382: 1346-1350.

5. Cammà C, Domenico MD, Monaco F (2011)Food Control Accepted

6. Dooley JJ, Paine KE, Garrett SD, Brown HM (2004) Detection of meat species using TaqMan real-time PCR assays.Meat Sci 68: 431-438.

7. Eijkelkamp JM, Aarts HJM, van der Fels-Klerx, van der HJ (2009) Suitability of Rapid Detection Methods for Salmonella. Food Analytical Methods 2: 1-13.

8. Elizaquível P, Aznar R (2008) A multiplex RTi-PCR reaction for simultaneous detection of Escherichia coli O157:H7, Salmonella spp. and Staphylococcus aureus on fresh, minimally processed vegetables.Food Microbiol 25: 705-713.

9. FDA, 2009. Guidance for Industry: Guide to Minimize Microbial Food Safety Hazards of Leafy Greens. online:http://www.fda.gov/Food/ GuidanceComplianceRegulatorylnformation/GuidanceDocuments/ ProduceandPlanProduct/ucm174200.htm (accessed 31 Jul 2009).

10. Guntupalli R, Lakshmanan RS, Hu J, Huang TS, Barbaree JM, et al. (2007) Rapid and sensitive magnetoelastic biosensors for the detection of Salmonella typhimurium in a mixed microbial population. J Microbiol Methods 70: 112-118.

11. Guo X, ChenJ, Beuchat LR, Brackett RE (2000) PCR Detection of Salmonella entericaSerotype Montevideo in and on Raw Tomatoes Using Primers Derived from hilA. Applied and Environmental Microbiology 66: 5248-5252.

12. Guo X, Chen J, Brackett RE, Beuchat LR (2001) Survival of Salmonellae on and in Tomato Plants from the Time of Inoculation at Flowering and Early Stages of Fruit Development through Fruit Ripening. Applied and Environmenta Microbiology 67: 4760-4764.

13. Huang S, Yang H, Lakshmanan RS, Johnson ML, Wan J Petrenko (2009) Bioelectron Sequential detection of Salmonella typhimurium and Bacillus anthracis spores using magnetoelastic Biosens Biosensors 24: 1730-1736.

14. Heller LC, Davis CR, Peak KK, Wingfield D, Cannons AC, et al. ( 2003 ) Comparison of methods for DNA isolation from food samples for detection of Shiga toxin-producing Escherichia coli by real-time PCR. Appl and Environ Microbiol 69: 1844-1846

15. Jung SJ, Kim HJ, kim HY (2005) Quantitative detection of Salmonella typhimurium contamination in milk, using real-time PCR. Journal of Microbiology and Biotechnology 15: 1353-1358.

16. Kesmen Z, Gulluce A, Sahin F, Yetim H (2009) Identification of meat species by TaqMan-based real-time PCR assay. Meat Sci 82: 444-449.

17. Lakshmanan RS, Guntupalli R, Hu J, Petrenko VA, Barbaree JM, et al. (2007) 
Citation: Park MK, Park JW, Wikle HC III, Chin BA (2012) Comparison of Phage-Based Magnetoelastic Biosensors with Taqman-Based Quantitative Real-Time PCR for the Detection of Salmonella typhimurium Directly Grown on Tomato Surfaces. J Biosens Bioelectron 3:113. doi:10.4172/2155-6210.1000113

Detection of Salmonella typhimurium in fat free milk using a phage immobilized magnetoelastic sensor. Sens Actuators B Chem 126: 544-550.

18. Li S, Li Y, Chen H, Horikawa S, Shen W, et al. (2010) Direct detection of Salmonella typhimurium on fresh produce using phage-based magnetoelastic biosensors. Biosens Bioelectron 26: 1313-1319.

19. Liming SH, Bhagwat AA (2004) Application of molecular beacon -- realtime PCR technology to detect Salmonella species contaminating fruits and vegetables. Int J Food Microbiol 95: 177-187.

20. Ma M, Michailides TJ (2007) Approaches for eliminating PCR inhibitors and designing PCR primers for the detection of phytopathogenic fungi. Crop Protection 26: 145-161.

21. Myint MS, Johnson YJ, Tablante NL, Heckert RA (2006) The effect of preenrichment protocol on the sensitivity and specificity of pcr for detection of naturally contaminated salmonella in raw poultry compared to conventional culture. Food microbiology 23: 599-604.

22. Park HJ, Kim HJ, Park SH, Shin EG, Kim HH, et al. (2008) Direct And Quantitative Analysis Of Salmonella Enterica Serovar Typhimurium Using RealTime Pcr From Artificially Contaminated Chicken Meat. J microbiol biotechnol 18: $1453-1458$.

23. Park MK, Li S, Chin BA (2011) Food and Bioprocess Technology In press.

24. Park MK, OH JH, Chin BA (2011) Sensors and Actuators B In press.
25. Perelle S, Dilasser F, Malorny B, Grout J, Hoorfar J, et al. (2004) Comparison of PCR-ELISA and LightCycler real-time PCR assays for detecting Salmonella spp. in milk and meat samples. Mol Cell Probes 18: 409-420.

26. Rudi K, Moen B, Drømtorp SM, Holck AL (2005) Use of ethidium monoazide and PCR in combination for quantification of viable and dead cells in complex samples. Applied And Environmental Microbiology 71: 1018-1024.

27. Shen W, Lakshmanan RS, Mathison LC, Petrenko VA, Chin BA (2009) Phage coated magnetoelastic micro-biosensors for real-time detection of Bacillus anthracis spores. Sens Actuators B Chem 137: 501-506.

28. Sorokulova IB, Olsen EV, Chen IH, Fiebor B, Barbaree JM, et al. (2005) Landscape phage probes for Salmonella typhimurium. J Microbiol Methods 63 $55-72$

29. Uyttendaele M, Vanwildemeersch K, Debevere J (2003) Evaluation of real-time PCR vs automated ELISA and a conventional culture method using a semisolid medium for detection of Salmonella. Lett Appl Microbiol 37: 386-391.

30. Velusamy V, Arshak K, Korostynska O, Oliwa K, Adley C (2010) Biotechnology Advances 28: 232-254

31. Wei Cl, Huang TS, Kim JM, Lin WF, Tamplin ML, Bartz JA (1995) Growth and survival of Salmonella montevideo on tomatoes and disinfection with chlorinated water. Journal of Food Protection 58: 829-836. 